\title{
ETS rearrangements and prostate cancer initiation
}

\author{
Arising from: Tomlins et al. Nature 448, 595-599 (2007)
}

The first recurrent translocation event in prostate cancer has been recently described ${ }^{1}$; it results in the translocation of an ETS (E26 transformation specific) transcription factor (ERG or ETV1) to the TMPRSS2 promoter region, which contains androgen responsive elements ${ }^{1}$. The TMPRSS2:ERG genetic rearrangement has been reported to occur in approximately $40 \%$ of primary prostate tumours (ETV1 genetic rearrangements occur at a much lower frequency), and it results in the aberrant androgen-regulated expression of $\mathrm{ERG}^{1-3}$. Tomlins et al. ${ }^{4}$ concluded that ETS genetic rearrangements are sufficient to initiate prostate neoplasia. However, here we show that ETS genetic rearrangements may in fact represent progression events rather than initiation events in prostate tumorigenesis. To this end, we demonstrate that the prostate-specific overexpression of ERG does not initiate prostate tumorigenesis.

We have found that mice overexpressing ERG (expression confirmed by quantitative PCR with reverse transcription (qRT-PCR), western blotting and immunohistochemistry) under the control of the probasin promoter (ARR2Pb, B6J background strain) do not develop neoplasia, and show only a very subtle phenotype of nuclear atypia (prominent nucleoli) without an increase in cellular proliferation (Fig. 1a). This is similar to what was shown by Tomlins et al. in their description of ETV1 transgenic mice 4 . These subtle nuclear changes without an increase in cellular layers are not sufficient to be classified as prostatic intraepithelial neoplasia (PIN), and are also frequently observed in the wild-type mouse prostate (Fig. 1a). We observe no notable differences in the prostate phenotype across all prostatic lobes (anterior, ventral and dorsal-lateral) between ERG transgenic and wild-type littermate mice. The subtle histological changes observed in ETV1 and ERG transgenic mice are markedly different from human a

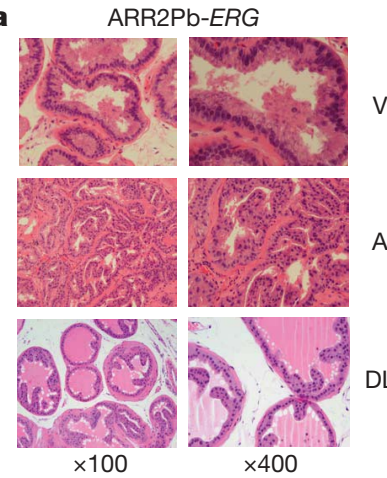

b Human HGPIN

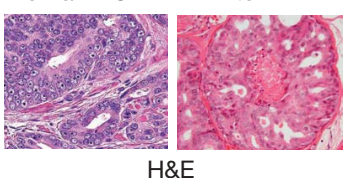

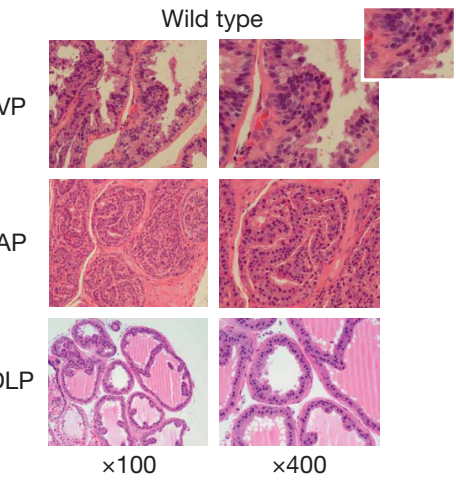

c ARR2Pb-ERG Wild type

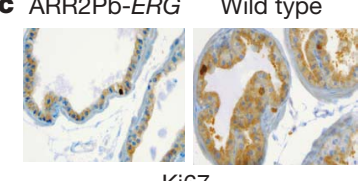

Ki67
Figure 1 | Prostate specific overexpression of ERG does not induce high grade prostatic intra-epithelial neoplasia. a, A total of 24 wild-type and $24 E R G$ transgenic mice were phenotypically characterized from one founding line after the establishment that three independent founding lines produced a similar phenotype. Low-power $(\times 100)$ and high-power $(\times 400)$ representative sections are shown for mice 6 months of age, demonstrating prominent nucleoli in wild-type mouse prostate glands. AP, anterior prostate lobes; DLP, dorsallateral prostate lobes; VP, ventral prostate lobes. $\mathbf{b}$, Representative histology of human HGPIN (left) and HGPIN in 12-month-old Pten heterozygous mice (right). H\&E, haematoxylin and eosin. c, Immunohistochemistry demonstrated no difference in Ki67 staining between wild-type and ERG transgenic mice. Original magnification in $b$ and $c, \times 400$. high-grade PIN (HGPIN) and from the HGPIN lesions that develop in Pten heterozygous mice (Fig. 1b). Furthermore, we did not observe an increase in the proliferative rate in the prostates of mice overexpressing $E R G$ compared to wild-type controls. As measured by Ki67 staining, on average $1 \%$ of the prostate epithelial cells in both ARR2Pb-ERG and wild-type mice were positive (Fig. 1c). With analyses at up to 18 months of age, no ARR2Pb-ERG mice have shown any change in phenotype. Whereas Tomlins et al. concluded that ETS genetic rearrangements are sufficient to initiate prostate neoplasia, we present data to suggest that ETS genetic rearrangements may in fact represent progression events rather than initiation events in prostate tumorigenesis, as there are no proliferative and pathological changes consistent with HGPIN found in either ERG or ETV1 mice.

Furthermore, the ERG translocation is infrequently found in human HGPIN and only in a minority (approximately 10-20\%) of patients who also have the translocation present in associated adenocarcinoma of the prostate. Most prostate cancer specimens with ERG genetic rearrangements do not show this rearrangement in the associated HGPIN. Therefore, the TMPRSS2:ERG translocation seems to be an early event in human prostate tumorigenesis, but one associated with progression from HGPIN to cancer.

Using mouse modelling, we have demonstrated that the aberrant expression of ERG is not sufficient to initiate neoplastic transformation but instead may cooperate with other genetic events to promote prostate cancer progression. We propose a working model whereby genetic initiating events conferring a proliferative advantage select for cooperating ETS genetic rearrangements that promote an invasive phenotype.

\section{METHODS}

Mice (B6J background strain) expressing ERG under the control of the probasin promoter $(\mathrm{ARR} 2 \mathrm{~Pb})$ were generated, genotyped and examined for transgene expression by qRT-PCR, western blotting and immunohistochemistry. We generated and analysed three independent $E R G$ lines. Founders were subsequently bred and four mice of each genotype were euthanized at 2, 4, 6, 8, 12 and 18 months of age. Prostate tissues were procured for formalin fixation, paraffin embedding and frozen storage for future molecular analyses.

Brett S. Carver ${ }^{1,3}$, Jennifer Tran', Zhenbang Chen ${ }^{1,5}$,

Arkaitz Carracedo-Perez ${ }^{1}$, Andrea Alimonti ${ }^{1,5}$, Caterina Nardella ${ }^{1,5}$, Anuradha Gopalan ${ }^{2}$, Peter T. Scardino ${ }^{3}$, Carlos Cordon-Cardo ${ }^{4}$, William Gerald ${ }^{2}$ \& Pier Paolo Pandolfi ${ }^{1,2,5}$

${ }^{1}$ Cancer Biology and Genetics Program, Sloan-Kettering Institute, Memorial Sloan-Kettering Cancer Center, New York, New York 10021, USA.

e-mail: ppandolf@bidmc.harvard.edu

${ }^{2}$ Department of Pathology, Memorial Sloan-Kettering Cancer Center, New York, New York 10021, USA.

${ }^{3}$ Department of Surgery, Division of Urology, Memorial Sloan-Kettering Cancer Center, New York, New York 10021, USA.

${ }^{4}$ Department of Pathology, Columbia University, New York, New York 10032, USA.

${ }^{5}$ Cancer Genetics Program, Beth Israel Deaconess Cancer Center and Department of Medicine, Beth Israel Deaconess Medical Center, Harvard Medical School, Boston, Massachusetts 02215, USA.

Received 19 March; accepted 20 November 2008.

1. Tomlins, S. A. et al. Recurrent fusion of TMPRSS2 and ETS transcription factor genes in prostate cancer. Science 310, 644-648 (2005).

2. Tu J. J., Rohan S., Kao J., Kitabayashi N., Mathew S. \& Chen Y.T.. Gene fusions between TMPRSS2 and ETS family genes in prostate cancer: frequency and transcript variant analysis by RT-PCR and FISH on paraffin-embedded tissues. Mod. Pathol. 20, 921-928 (2007)

3. Perner, S. et al. TMPRSS2-ERG fusion prostate cancer: an early molecular event associated with invasion. Am. J. Surg. Pathol. 31, 882-888 (2007)

4. Tomlins, S. A. et al. Distinct classes of chromosomal rearrangements create oncogenic ETS gene fusions in prostate cancer. Nature 448, 595-599 (2007)

doi:10.1038/nature07738 


\section{Tomlins et al. reply}

Replying to: Carver, B. S. et al. Nature 457, doi:10.1038/nature07738 (2009)

Carver et al. ${ }^{1}$ question our recent report that mice expressing ETV1 under the control of the probasin promoter $(\mathrm{ARR} 2 \mathrm{~Pb})$ develop mouse prostatic intraepithelial neoplasia $(\mathrm{mPIN})^{2}$. They report the generation of transgenic ARR2Pb-ERG mice with no phenotypic differences from control mice. They propose that this demonstrates that ETS genetic rearrangements do not initiate prostate tumorigenesis and use data from human prostate cancer studies to propose that ETS rearrangements are associated with progression from PIN to prostate cancer. Although we and others have shown that ARR2Pb-ETV1 and ARR2Pb-ERG mice develop mPIN, we have consistently proposed that in human prostate cancer development, ETS rearrangements mediate the transition from PIN to cancer.

Our blinded histopathological evaluation of the ARR2Pb-ETV1 mice included two genitourinary pathologists (R.B.S. and M.A.R.), one of whom is a co-author on 'The consensus report from the Bar Harbor Meeting of the Mouse Models of Human Cancer Consortium Prostate Pathology Committee' (M.A.R.), and the diagnosis of mPIN without progression to carcinoma was made according to those criteria ${ }^{3}$.

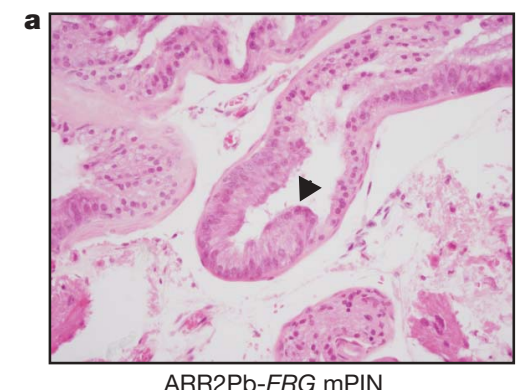

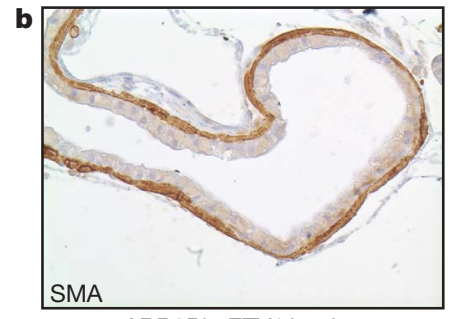

ARR2Pb-ETV1 benign

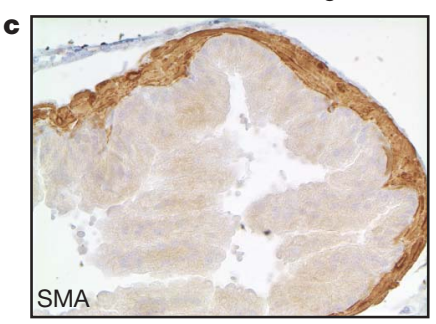

ARR2Pb-ETV1 mPIN

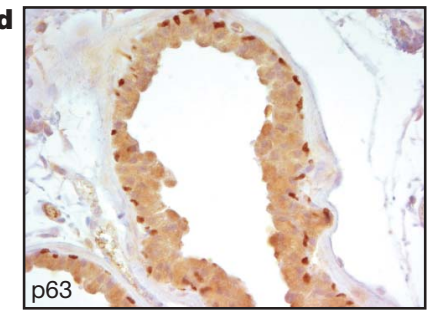

ARR2Pb-ETV1 benign

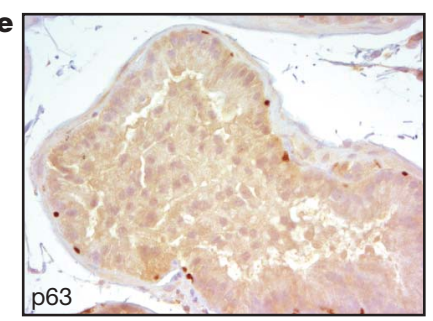

ARR2Pb-ETV1 mPIN
Figure 1 | $\mathrm{mPIN}$ in ARR2Pb-ERG and ARR2Pb-ETV1 mice. a, In ARR2Pb$E R G$ mice, distinct areas of proliferation in sporadic glands, consistent with the definition of mPIN (black arrowhead), were observed adjacent to normal prostatic epithelium. b-e, Loss of the circumferential basal layer in mPIN lesions in ARR2Pb-ETV1 mice. Consistent with the focal nature of mPIN, normal areas and mPIN were observed in the prostate of ARR2Pb-ETV1 mice. Immunohistochemistry with smooth muscle actin (SMA) demonstrates a continuous fibromuscular layer around benign glands (b) and all mPIN lesions (c), whereas the basal cell marker p63 demonstrates the loss of circumferential basal cells in MPIN foci (e) compared to normal glands (d) in the dorsolateral prostate of a ARR2Pb-ETV1 mouse. Original magnification for all images is $\times 400$.
Notably, members of the Bar Harbour Committee observed a wide spectrum of morphological alterations that were all considered mPIN, and the exact replication of human high-grade PIN is not required to define mPIN. In fact, the group of human and animal pathologists adopted this view to reflect the wide spectrum of variations observed between different mouse models of prostate cancer.

We also generated ARR2Pb-ERG mice, which again by the Bar Harbour Committee classification develop mPIN without progression to carcinoma (Fig. 1a). Similar to ARR2Pb-ETV1 mice, ARR2Pb-ERG mice have focal lesions showing nuclear atypia, including stratification, hyperchromasia and macronucleoli ${ }^{4}$. The development of $\mathrm{mPIN}$ without progression to carcinoma in ARR2Pb-ERG transgenic mice has also been previously described ${ }^{5}$. In this model, a decrease in basal epithelial cells was shown in mPIN lesions, and luminal epithelial cells directly contacted the stromal cell compartment ${ }^{5}$. We found a similar loss of the circumferential basal epithelial layer in MPIN lesions from our ARR2Pb-ETV1 (Fig. 1b-e) and ARR2Pb-ERG mice ${ }^{4}$, which is a hallmark of prostate carcinoma development in both mice and humans ${ }^{6}$. As our ARR2Pb-ERG mice and those used by Klezovitch et al. ${ }^{5}$ were generated on different backgrounds, this strongly supports a phenotypic effect in ARR2Pb-ERG mice. It is unclear whether Carver et al. looked for changes in the relationship between the basal epithelial layer and the stromal compartment in their model.

Although we feel that prostate specific expression of ERG or ETV1 induces PIN in mice, we have never claimed that this (or any other evidence) supports ETS rearrangements initiating human prostate cancer tumorigenesis through the development of PIN. Instead, through our results including ERG expression in human PIN and prostate cancer by DNA microarray analysis ${ }^{7}$, fluorescence in situ hybridization (FISH) data showing the frequency of ETS rearrangements in PIN and prostate cancer $^{8,9}$, ERG knockdown in VCaP (a TMPRSS2:ERG positive prostate cancer cell line) deregulating the transcriptional program differentiating PIN and prostate cancer ${ }^{4}$, in vitro data demonstrating a role for ETV1 and ERG in invasion ${ }^{2,4}$, and ARR2Pb-ERG and ARR2Pb-ETV1 mice not developing frank carcinoma ${ }^{2,4}$, we have consistently proposed that ETS gene fusions in humans mediate the transition from prostate cells with pre-existing lesions ( such as cells in PIN foci) to carcinoma ${ }^{2,4,7-10}$. We feel that models combining ETS rearrangements and other early lesions in prostate cancer development have the potential to transform in vitro and in vivo prostate cancer research.

Scott A. Tomlins ${ }^{1}$, Bharathi Laxman ${ }^{1}$, Saravana M. Dhanasekaran', Beth E. Helgeson ${ }^{1}$, Xuhong Cao', David S. Morris ${ }^{2}$, Anjana Menon', Xiaojun Jing ${ }^{1}$, Qi Cao ${ }^{1}$, Bo Han ${ }^{1}$, Jindan $\mathrm{Yu}^{1}$, Lei Wang ${ }^{1}$,

James E. Montie ${ }^{2,4}$, Mark A. Rubin ${ }^{5,6} \uparrow$, Kenneth J. Pienta ${ }^{2,3,4}$, Diane Roulston ${ }^{1}$, Rajal B. Shah ${ }^{1,2,4}$, Sooryanarayana Varambally ${ }^{1,4}$, Rohit Mehra $^{1,4}$ \& Arul M. Chinnaiyan ${ }^{1,2,4}$

${ }^{1}$ Michigan Center for Translational Pathology, Department of Pathology, University of Michigan Medical School, Ann Arbor, Michigan 48109,

USA.

e-mail: arul@umich.edu

${ }^{2}$ Department of Urology, University of Michigan Medical School, Ann

Arbor, Michigan 48109, USA.

${ }^{3}$ Department of Internal Medicine, University of Michigan Medical

School, Ann Arbor, Michigan 48109, USA.

${ }^{4}$ Comprehensive Cancer Center, University of Michigan Medical School, Ann Arbor, Michigan 48109, USA.

${ }^{5}$ Department of Pathology, Brigham and Women's Hospital, Harvard

Medical School, Boston, Massachusetts, 02115, USA.

${ }^{6}$ Dana-Farber Cancer Institute, Harvard Medical School, Boston,

Massachusetts, 02115, USA.

†Present address: Department of Pathology and Laboratory Medicine,

Cornell University, New York, New York 10021, USA. 
1. Carver, B. S. et al. ETS rearrangements and prostate cancer initiation. Nature 457, doi:10.1038/nature07738 (2009).

2. Tomlins, S. A. et al. Distinct classes of chromosomal rearrangements create oncogenic ETS gene fusions in prostate cancer. Nature 448, 595-599 (2007)

3. Shappell, S. B. et al. Prostate pathology of genetically engineered mice: definitions and classification. The consensus report from the Bar Harbor meeting of the Mouse Models of Human Cancer Consortium Prostate Pathology Committee. Cancer Res. 64, 2270-2305 (2004).

4. Tomlins, S. A. et al. Role of the TMPRSS2-ERG gene fusion in prostate cancer. Neoplasia 10, 177-188 (2008).

5. Klezovitch, O. et al. A causal role for ERG in neoplastic transformation of prostate epithelium. Proc. Natl Acad. Sci. USA 105, 2105-2110 (2008).
6. DeMarzo, A. M., Nelson, W. G., Isaacs, W. B. \& Epstein, J. I. Pathological and molecular aspects of prostate cancer. Lancet 361, 955-964 (2003).

7. Tomlins, S. A. et al. Integrative molecular concept modeling of prostate cancer progression. Nature Genet. 39, 41-51 (2007).

8. Mosquera, J. M. et al. Characterization of TMPRSS2-ERG fusion high-grade prostatic intraepithelial neoplasia and potential clinical implications. Clin. Cancer Res. 14, 3380-3385 (2008).

9. Perner, S. et al. TMPRSS2-ERG fusion prostate cancer: an early molecular event associated with invasion. Am. J. Surg. Pathol. 31, 882-888 (2007).

10. Kumar-Sinha, C., Tomlins, S. A. \& Chinnaiyan, A. M. Recurrent gene fusions in prostate cancer. Nature Rev. Cancer 8, 497-511 (2008).

doi:10.1038/nature07739 